\title{
A novel R2R3-MYB from grape hyacinth, MaMybA, which is different from MaAN2, confers intense and magenta anthocyanin pigmentation in tobacco
}

Kaili Chen ${ }^{1,2,3}$, Lingjuan Du ${ }^{1,2,3}$, Hongli Liu ${ }^{1,2,3}$ and Yali Liu $u^{1,2,3^{*}}$ (D)

\begin{abstract}
Background: The primary pigments in flowers are anthocyanins, the biosynthesis of which is mainly regulated by R2R3-MYBs. Muscari armeniacum is an ornamental garden plant with deep cobalt blue flowers containing delphinidin-based anthocyanins. An anthocyanin-related R2R3-MYB MaAN2 has previously been identified in M. armeniacum flowers; here, we also characterized a novel R2R3-MYB MaMybA, to determine its function and highlight similarities and differences between MaMybA and MaAN2.
\end{abstract}

Results: In this study, a novel anthocyanin-related R2R3-MYB gene was isolated from M. armeniacum flowers and functionally identified. A sequence alignment showed that MaMybA contained motifs typically conserved with MaAN2 and its orthologs. However, the shared identity of the entire amino acid sequence between MaMybA and MaAN2 was 43.5\%. Phylogenetic analysis showed that they were both clustered into the AN2 subgroup of the R2R3-MYB family, but not in the same branch. We also identified a Illf bHLH protein, MabHLH1, in M. armeniacum flowers. A bimolecular fluorescence complementation assay showed that MabHLH1 interacted with MaMybA or MaAN2 in vivo; a dual luciferase assay indicated that MaMybA alone or in interaction with MabHLH1 could regulate the expression of MaDFR and AtDFR, but MaAN2 required MabHLH1 to do so. When overexpressing MaMybA in Nicotiana tabacum 'NC89', the leaves, petals, anthers, and calyx of transgenic tobacco showed intense and magenta anthocyanin pigments, whereas those of OE-MaAN2 plants had lighter pigmentation. However, the ovary wall and seed skin of OE-MaMybA tobacco were barely pigmented, while those of OE-MaAN2 tobacco were reddish-purple. Moreover, overexpressing MaMybA in tobacco obviously improved anthocyanin pigmentation, compared to the OEMaAN2 and control plants, by largely upregulating anthocyanin biosynthetic and endogenous bHLH genes. Notably, the increased transcription of NtF3'5'H in OE-MaMybA tobacco might lead to additional accumulation of delphinidin 3-rutinoside, which was barely detected in OE-MaAN2 and control plants. We concluded that the high concentration of anthocyanin and the newly produced Dp3R caused the darker color of OE-MaMybA compared to OE-MaAN2 tobacco.

Conclusion: The newly identified R2R3-MYB transcription factor MaMybA functions in anthocyanin biosynthesis, but has some differences from MaAN2; MaMybA could also be useful in modifying flower color in ornamental plants.

Keywords: Anthocyanin biosynthesis, Flower color, Monocot plants, R2R3-MYB, Transcription regulation, Transgenic tobacco

\footnotetext{
*Correspondence: Iyl6151@126.com

${ }^{1}$ College of Landscape Architecture and Arts, Northwest A\&F University, Yangling 712100, Shaanxi, People's Republic of China

${ }^{2}$ Key Laboratory of Horticultural Plant Biology and Germplasm Innovation in Northwest China, Ministry of Agriculture, Yangling 712100, Shaanxi, People's Republic of China

Full list of author information is available at the end of the article
}

(c) The Author(s). 2019 Open Access This article is distributed under the terms of the Creative Commons Attribution 4.0 International License (http://creativecommons.org/licenses/by/4.0/), which permits unrestricted use, distribution, and reproduction in any medium, provided you give appropriate credit to the original author(s) and the source, provide a link to the Creative Commons license, and indicate if changes were made. The Creative Commons Public Domain Dedication waiver (http://creativecommons.org/publicdomain/zero/1.0/) applies to the data made available in this article, unless otherwise stated. 


\section{Background}

A desirable floral color is a typical trait of ornamental plants [1]; it is mainly attributable to anthocyanins, carotenoids, and betalains [2]. Anthocyanins are the primary pigments in flowers, fruit, and other plant tissues, imparting a wide range of colors, from red to violet/blue [3]. They also function in flower pollination, enhancing the tolerance of plants to biological and abiotic stress. Moreover, they are important in the food industry, where they are used as safe and natural colorants [2]. The anthocyanin biosynthetic pathway, in which anthocyanin biosynthetic genes and their regulators are essential to producing anthocyanins, has been the subject of extensive and thorough study [4]. In recent years, genetic engineering has proven to be a promising approach to flower color modification [5]; in ornamental plants, the main approach has been molecular breeding through genetic regulation of anthocyanin synthesis [6]. Although there are many examples of successful flower modification engineering in various ornamental species, either by overexpressing or silencing of diverse anthocyanin biosynthetic genes, such as in gerbera [7], rose [4], carnation [8], and chrysanthemum [9, 10], regulatory proteins may also offer considerable potential in controlling anthocyanin biosynthesis.

Anthocyanin biosynthetic regulators include R2R3MYB transcription factors (TFs), their partners the basic helix-loop-helix (bHLH) proteins, and WD-repeat (WDR) proteins; together, these form the MYB-bHLHWDR (MBW) complex, in which R2R3-MYBs, which regulate anthocyanin biosynthesis, are the key TFs determining the intensity and patterning of pigmentation in plants [8]. There have been numerous studies of R2R3MYBs in both dicot and monocot ornamental plants. In dicot ornamental plants, for example, AmRosea1, AmRosea2, and Amvenosa in Antirrhinum majus have been shown to control anthocyanin accumulation in different flower parts [11]. In Lisianthus, overexpression of AmRosea1 leads to an increased level of anthocyanins and a changed color in the petals and sepals of transgenic plants [12]. Additionally, expression of both AmRosea 1 and AmDelila (which encode a bHLH protein related to anthocyanin pigmentation in snapdragon flowers) has been shown to induce a high concentration of delphinidin- (Dp-) based anthocyanins in the peel and flesh of transgenic tomato fruits [13]. GMYB10, an anthocyanin-promoting R2R3-MYB in Gerbera hybrida, was overexpressed in the Gerbera cultivar 'Terra Regina' (pelargonidin-based flowers), obviously enhancing the anthocyanin level, and inducing novel cyanidin (Cy) biosynthesis [7, 14]. In monocot flowers, Lilium LhMYB12Lat [15], Anthurium andraeanum AaMYB2 [16], and Muscari armeniacum MaAN2 [17] have been shown to promote anthocyanin accumulation in tobacco plants. In the model plant Arabidopsis thaliana, PAP1 and PAP2 increased anthocyanin accumulation when overexpressing as transgenes in Arabidopsis, tobacco, and Rosa hybrida [18-20]. Therefore, identifying these anthocyanin regulators is meaningful in molecular breeding aimed at modifying flower color in ornamental plants, and will even be useful in the commercial production of anthocyanins for use as colorings in the food industry.

Grape hyacinth (M. armeniacum) is a species of perennial bulbous monocotyledonous plants that blossom in mid-spring, and is often used as a garden ornamental. Its flowers are a naturally deep cobalt blue color, containing Dp-based anthocyanins [21, 22], and it provides suitable material for the study of blue flower coloration in monocots [23]. In our previous study, we only screened one anthocyanin-related R2R2-MYB unigene by functional annotation in the transcriptome of $M$. armeniacum flowers [23], and then named MaAN2, which was identified that it could induce anthocyanin accumulation in tobacco, and interacted with a bHLH protein, AtTT8, to regulate the expression of anthocyanin late biosynthetic genes (LBGs; i.e., MaDFR and $M a A N S)$ [17] . In the present study, we excavated a novel anthocyanin-related R2R3-MYB TF named MaMybA, using a typical anthocyanin-regulator AtPAP1 from the $M$. armeniacum flowers transcriptome [23], by local BLASTP (BLAST protein database). We found that MaMybA differed from MaAN2, and we further verified the function of $M a M y b A$ in anthocyanin biosynthesis by heterologous expression in tobacco. The study aimed to determine the regulatory mechanism that governs coloration in $M$. armeniacum flowers, identify a valuable anthocyanin-promoting regulatory gene for use in the modification of flower colors in ornamental plants, and determine similarities and differences between MaMybA and MaAN2 in anthocyanin synthesis.

\section{Results}

Sequence alignment and phylogenetic analysis of R2R3-MYB MaMybA isolated from M. armeniacum

One R2R3-MYB unigene was screened with local BLASTP, using anthocyanin-related R2R3-MYB TF AtPAP1 from an M. armeniacum flower transcriptome [23]. Its full-length cDNA sequence was obtained by RACE-PCR. The $711 \mathrm{bp}$ open reading frame (ORF) of MaMybA encoded 237 amino acids. The cDNA sequence was submitted to the NCBI GenBank (accession number MF663728).

Sequence alignment showed that the highly conserved MYB domain (i.e., the R2R3 repeats) is present in the $\mathrm{N}$ terminal of MaMybA, MaAN2, and other anthocyaninrelated R2R3-MYBs (Fig. 1a). In addition, a conserved motif $[\mathrm{D} / \mathrm{E}] \mathrm{LX}_{2}[\mathrm{R} / \mathrm{K}] \mathrm{X}_{3} \mathrm{LX}_{6} \mathrm{LX}_{3} \mathrm{R}$, which is necessary to the interaction of the MYB domain with the R-like 


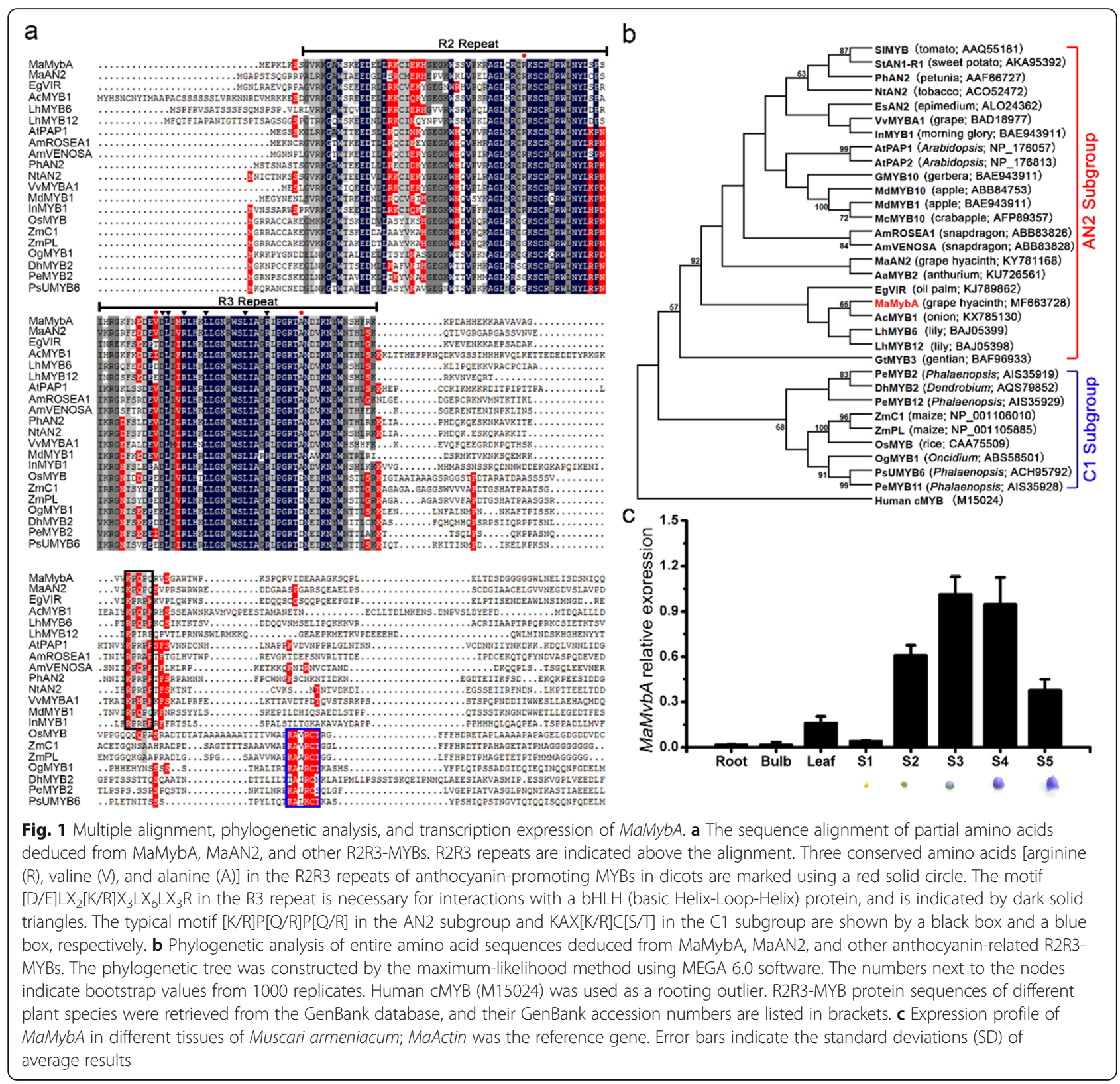

bHLH proteins [24], was present in the R3 repeat of MaMybA, MaAN2, and other anthocyanin-related R2R3-MYBs (Fig. 1a). When considering the entire amino acid sequence, MaMybA showed only $43.5 \%$ shared identity with MaAN2, 47.3\% shared identity with EgVIR (an R2R3-MYB controlling seed color in Elaeis guineensis [25], and 43.07\% shared identity with AcMYB1 (an R2R3-MYB regulating the anthocyanin accumulation of bulbs in Allium cepa) [26] (Fig. 1a). Moreover, MaMybA and MaAN2 shared three conserved amino acids [Arg (R), Val (V), and Ala (A)] in the $\mathrm{R} 2 \mathrm{R} 3$ repeats, and a highly conserved motif $[\mathrm{K} / \mathrm{R}] \mathrm{P}[\mathrm{Q} /$ $\mathrm{R}] \mathrm{P}[\mathrm{Q} / \mathrm{R}]$ in the $\mathrm{C}$-terminal with MYBs in the AN2 subgroup from eudicots and some monocots (Fig. 1a).
A phylogenetic analysis indicated that MaMybA, along with MaAN2, AtPAP1, LhMYB6, and AcMYB1, was clustered into the AN2 subgroup, and was most closely associated with AcMYB1 (Fig. 1b). However, MYBs from the monocot families Poaceae and Orchidaceae were grouped into the $\mathrm{C} 1$ subgroup (Fig. 1b). Additionally, the relative expression of $M a M y b A$ in the roots, bulbs, leaves, and five different floral developmental stages (S1 S5) showed that MaMybA was predominantly expressed in pigmented flowers. The mRNA level of $M a M y b A$ was very low in the roots, bulbs, and flowers of stage S1, which was much lower than in the leaves. The $M a M y b A$ transcript in flowers gradually increased, peaking at stage $S 3$, and then 
slightly decreased from stages S3 to S4, before showing a fall by stage S5 (Fig. 1c).

\section{MaMybA was localized in the cell nucleus and had transcriptional activation ability}

In order to detect the subcellular localization of MaMybA, the plasmids 35S:GFP (Green fluorescent protein) and MaMybA-GFP were separately cotransformed with AtHY5-(ELONGATED HYPOCOTYL5; AT5G11260) mCherry into Arabidopsis mesophyll protoplasts. The GFP fluorescence of MaMybA was visualized to overlap with the mCherry fluorescence of AtHY5, which is a known nuclear protein [27], indicating that MaMybA was localized in the nuclei of $A$. thaliana protoplasts (Fig. 2).

We performed a transactivation assay in yeast in order to assess the transcriptional activation ability of MaMybA. As shown in Additional file 1: Figure S1, the yeasts only transformed with the positive control (pGBKT7-53 + pGADT7-T), and the pGBKT7/MaMybA plasmid was able to grow in SD/-Trp media to which were added $40 \mu \mathrm{g} \mathrm{mL}^{-1} \mathrm{X}$ - $\alpha$-gal and $200 \mathrm{ng} \mathrm{mL}^{-1}$ aureobasidin A (AbA), and exhibited blue plaques. However, the negative control was not able to grow in SD/-Trp media plus $200 \mathrm{ng} \mathrm{mL}^{-1}$ AbA or $40 \mu \mathrm{g} \mathrm{mL}^{-1} \mathrm{X}$ - $\alpha$-gal and $200 \mathrm{ng} \mathrm{mL}^{-1}$ AbA. The results showed that MaMybA has transcriptional activation ability.

\section{MabHLH1 interacted with MaMybA or MaAN2 in vivo}

R2R3-MYBs have been reported to interact with the IIIf bHLH protein that regulates anthocyanin biosynthesis [28-30]. We screened one anthocyanin-related bHLH unigene from the transcriptome of $M$. armeniacum with local BLASTP, using the R-like IIIf bHLH protein AtTT8. A $2001 \mathrm{bp}$ full-length cDNA was obtained from $M$. armeniacum flowers using a reverse transcriptionpolymerase chain reaction (RT-PCR), and named MabHLH1 (MF663729). MabHLH1 contained a highly conserved MYB interaction region in the N-terminal, and a bHLH DNA binding domain in the C-terminal (Additional file 2: Figure S2a). The phylogenetic analysis showed that MabHLH1 was grouped with LhbHLH1

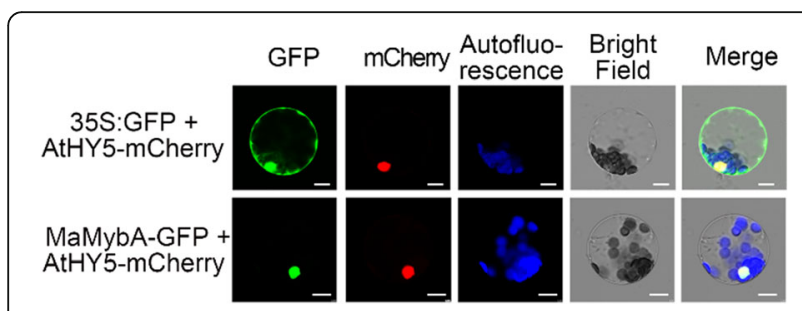

Fig. 2 Subcellular localization of MaMybA. The transient coexpression of MaMybA-GFP with AtHY5-mCherry in Arabidopsis thaliana mesophyll protoplasts showed that MaMybA and AtHY5 were co-located in the nucleus. Bars: $10 \mu \mathrm{m}$ and ZmLC into the LC/JAF13/DEL clade of the IIIf subgroup of the flavonoid-related bHLH proteins (Additional file 2: Figure S2b), and was also constitutively expressed in different tissues of $M$. armeniacum (Additional file 2: Fig. S2c). Subcellular localization and transcriptional activation ability assays showed that MabHLH1 was localized in the nucleus and had transactivation ability, thus meaning it was a TF (Additional file 3: Figure S3). A bimolecular fluorescence complementation (BiFC) assay was then conducted to verify the interaction between MabHLH1 and MaMybA or MaAN2 in vivo (Fig. 3a). Co-expressing either $\mathrm{YC} /$ MaMybA with YN/MabHLH1 or YC/MabHLH1 with YN/MaAN2 in Nicotiana benthamiana leaves revealed yellow fluorescent protein (YFP) fluorescence in the epidermal cell nuclei (Fig. 3a). Thus, MabHLH1 was shown to be an interacting protein from the anthocyanin-related R2R3-MYBs in M. armeniacum, which was able to interact with MaMybA or MaAN2 in vivo.

\section{MaMybA activated the anthocyanin biosynthetic gene promoters}

In order to investigate whether MaMybA or MabHLH1 is able to control expression of the anthocyanin biosynthetic genes, a dual-luciferase assay was performed on $N$. benthamiana leaves (Fig. 3b). The promoters of the anthocyanin early biosynthetic gene (EBG) MaCHS (M. armeniacum; KY781171) and the key LBGs, AtDFR (A. thaliana; AT5G42800) and MaDFR (M. armeniacum; KY781169), were fused to LUC (firefly luciferase). $M a M y b A, M a A N 2$, and MabHLH1 were driven by $35 \mathrm{~S}$ promoters. Each or both of the TFs were co-infiltrated with each promoter. The results showed that single or both of the TFs could not increase the promoter activity of MaCHS. However, MaMybA alone could activate the $M a D F R$ and AtDFR promoters, while neither MaAN2 nor MabHLH1 were able to activate both. When co-expressing MaMybA with MabHLH1, the promoter activities of $M a D F R$ and AtDFR were significantly promoted, by 1.6 and 1.1 times higher than when infiltrated with MaMybA alone, respectively (Fig. 3b). When co-infiltrating MaAN2 with MabHLH1, the promoter activities of MaDFR and AtDFR were also significantly promoted, 4.0 and 22.2 times higher, when compared with MaAN2 infiltrated alone, respectively (Fig. 3b). Therefore, the results indicated that, MaMybA alone or in interaction with MabHLH1, could control the transcription of MaDFR, but MaAN2 required a direct interaction with MabHLH1 to do so.

Heterologous expression of MaMybA in tobacco strongly promoted anthocyanin accumulation

In order to characterize the function of $M a M y b A$, $M a M y b A$ driven by a $35 \mathrm{~S}$ promoter was transformed 


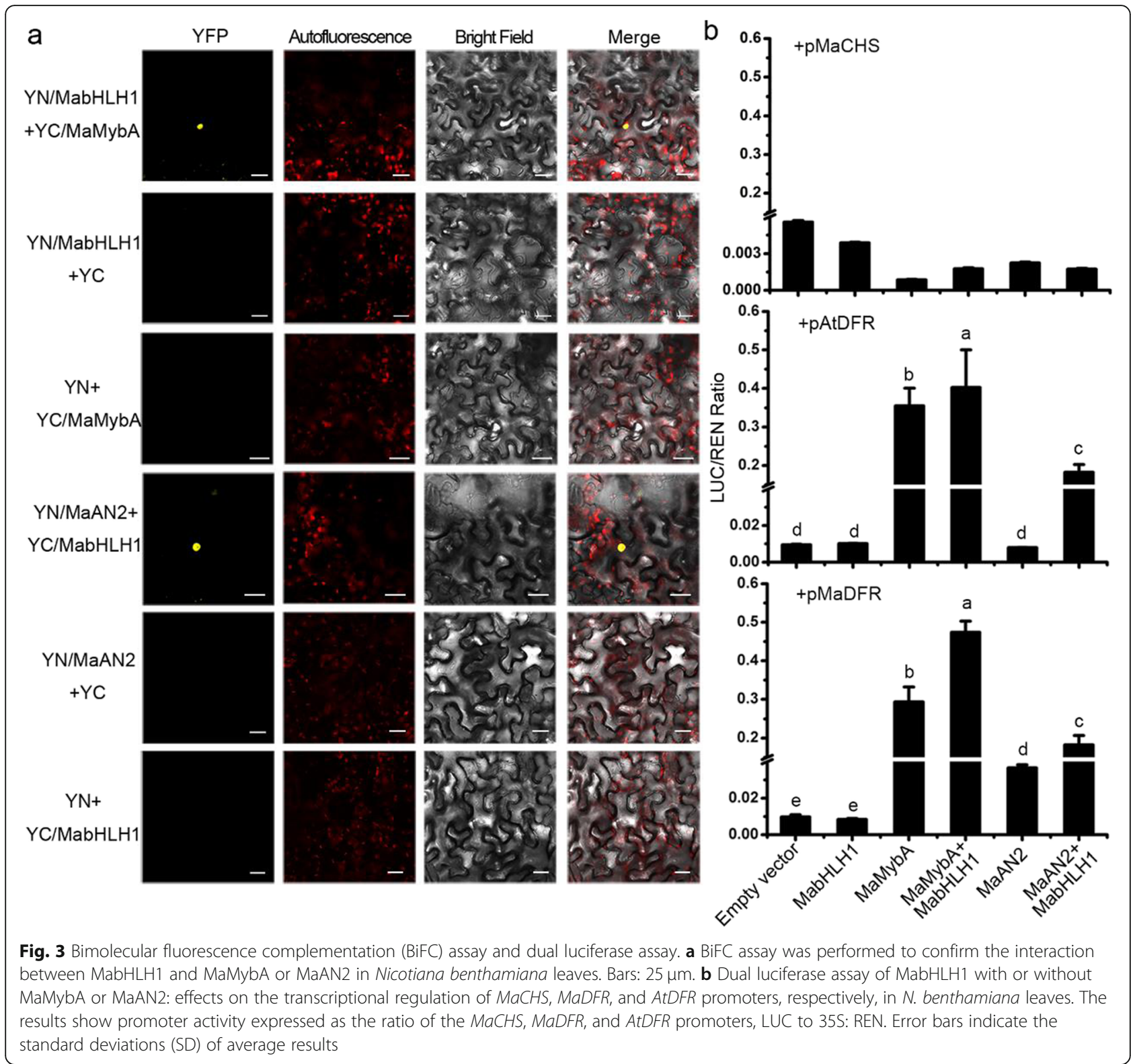

into tobacco ( $N$. tabacum ' $\mathrm{NC} 89$ ') leaf discs by an Agrobacterium-mediated transformation. Three $\mathrm{T}_{1}$ overexpressing MaMybA (OE-MaMybA) transgenic lines, designated as $\mathrm{OE} \# 1, \mathrm{OE} \# 2$, and $\mathrm{OE} \# 3$, were generated (Fig. 4a, b). Additionally, the $\mathrm{T}_{1}$ overexpressing MaAN2 tobacco (the most pigmentation line (i.e., line 2) among three OE-MaAN2 transgenic plants in respect to Chen et al. [17]) was used as the positive control (OEMaAN2), and the empty vector transgenic plant was used as the negative control (control). The results showed that MaMybA strongly increased anthocyanin production and conferred an intense red-purple color in tobacco, especially in the leaf, calyx, petal, and anther (Fig. 4a-c), while the ovary wall and seed skin were barely anthocyanin pigmentation (Fig. 4c, d). However,
OE-MaAN2 tobacco presented a much lighter magenta color in the leaf, calyx, and anther than did OE$M a M y b A$ tobacco; however, the ovary wall and seed skin were both pigmented with a reddish color, and the corolla was deep pink (Fig. 4a-d).

Floral color is related to the internal or surface tissue morphology of floral organs, and to the type and content of anthocyanin pigments in the floral cells; pigment, however, is the key determinant [1]. We first determined the content and type of anthocyanin in tobaccos using high-performance liquid chromatography (HPLC). The anthocyanin concentration in the leaves and corollas of three OE-MaMybA tobaccos was obviously higher than in the OE-MaAN2 and control tobaccos (Fig. 4e, f). Specifically, the anthocyanin level in three lines of OE- 

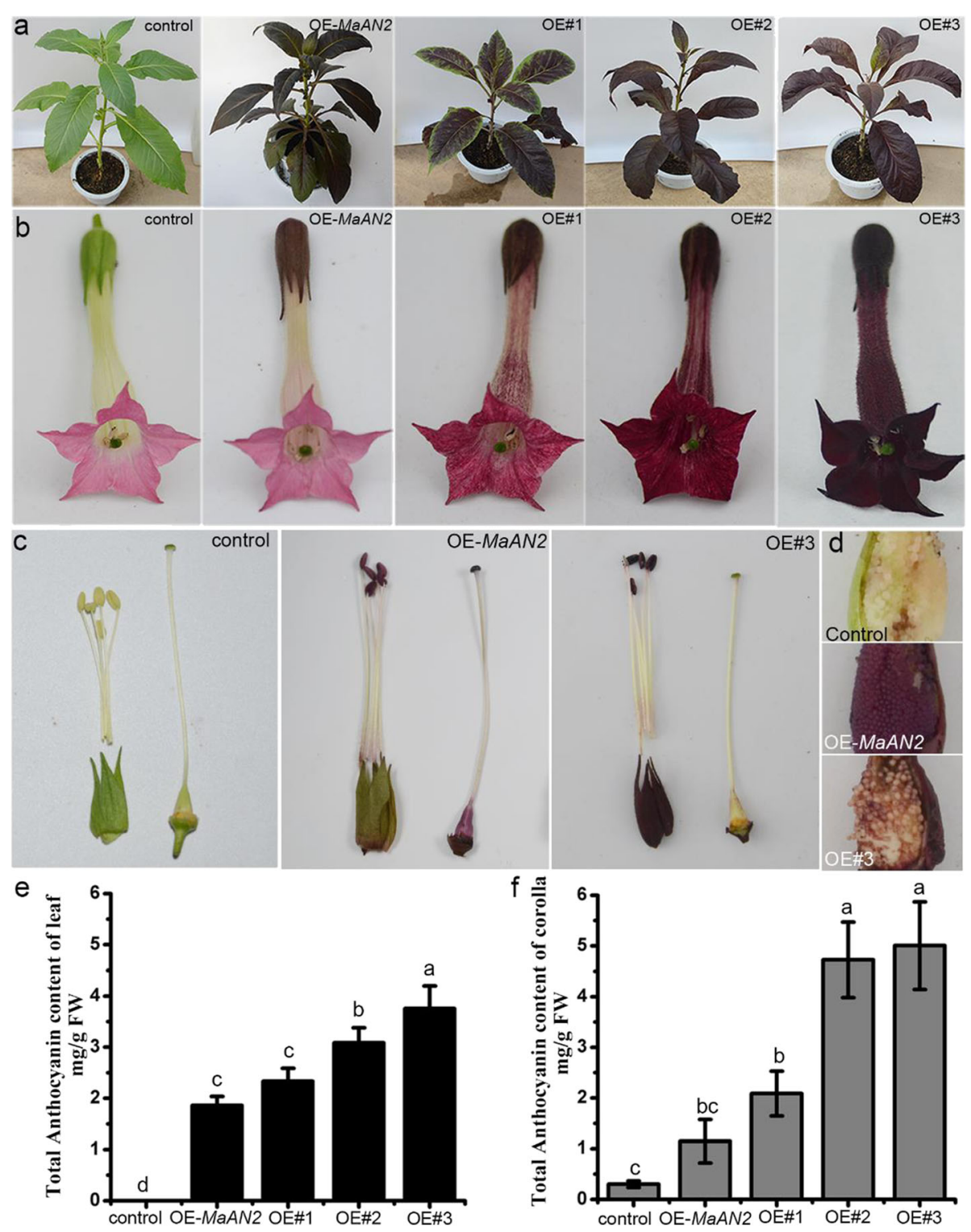

Fig. 4 Phenotypic observation and total anthocyanin content of the leaves and corollas of the control, OE (overexpressing)-MaAN2, and three OE-MaMybA line (OE\#1, OE\#2, and OE\#3) tobaccos. Phenotype of total plant (a), phenotype of flower (b), calyx, anthers, filament, and ovary of a flower $(\mathbf{c})$, and seeds $(\mathbf{d})$. A quantitative determination of the anthocyanin content of the leaves (e) and corollas (f) in the control, OE-MaAN2, and three OE-MaMybA line tobaccos. The anthocyanin extracts from the leaves and corollas were analyzed using high-performance liquid chromatography and were each monitored at $530 \mathrm{~nm}$. A standard curve of cyanidin 3-rutinoside content was used as a control to calculate the anthocyanin content in the tobacco. Error bars indicate standard deviations (SD) in the average anthocyanin content

$M a M y b A$ leaves was 1.25 - to 2-fold higher than that of OE-MaAN2 leaves. The level of anthocyanin in three lines of OE-MaMybA corollas was 1.8- to 4.3-fold higher than that of OE-MaAN2 corollas, and was 6.9- to 16.7fold higher than that of the control corollas (Fig. 4e, f). Anthocyanins were virtually undetectable in the control tobacco leaves (Fig. 4e, Fig. 5a, Additional file 4: Figure S4). Next, we detected two main anthocyanins (peaks 1 and 2) in the leaves and corollas of three OE-MaMybA lines
(OE\#3 as the representative in Fig. 5a; Additional file 4: Figure S4), while primarily a single anthocyanin (peak 2) was detected in the corollas of the control and three OEMaAN2 lines mentioned in Chen et al. [17] (line 2 as the representative in Fig. 5a, Additional file 4: Figure S4).

Previous studies have shown the primary anthocyanin in tobacco to be cyanidin 3-rutinoside (Cy3R) [31]; the retention times of the delphinidin 3-rutinoside (Dp3R) and Cy3R standards were very close to 

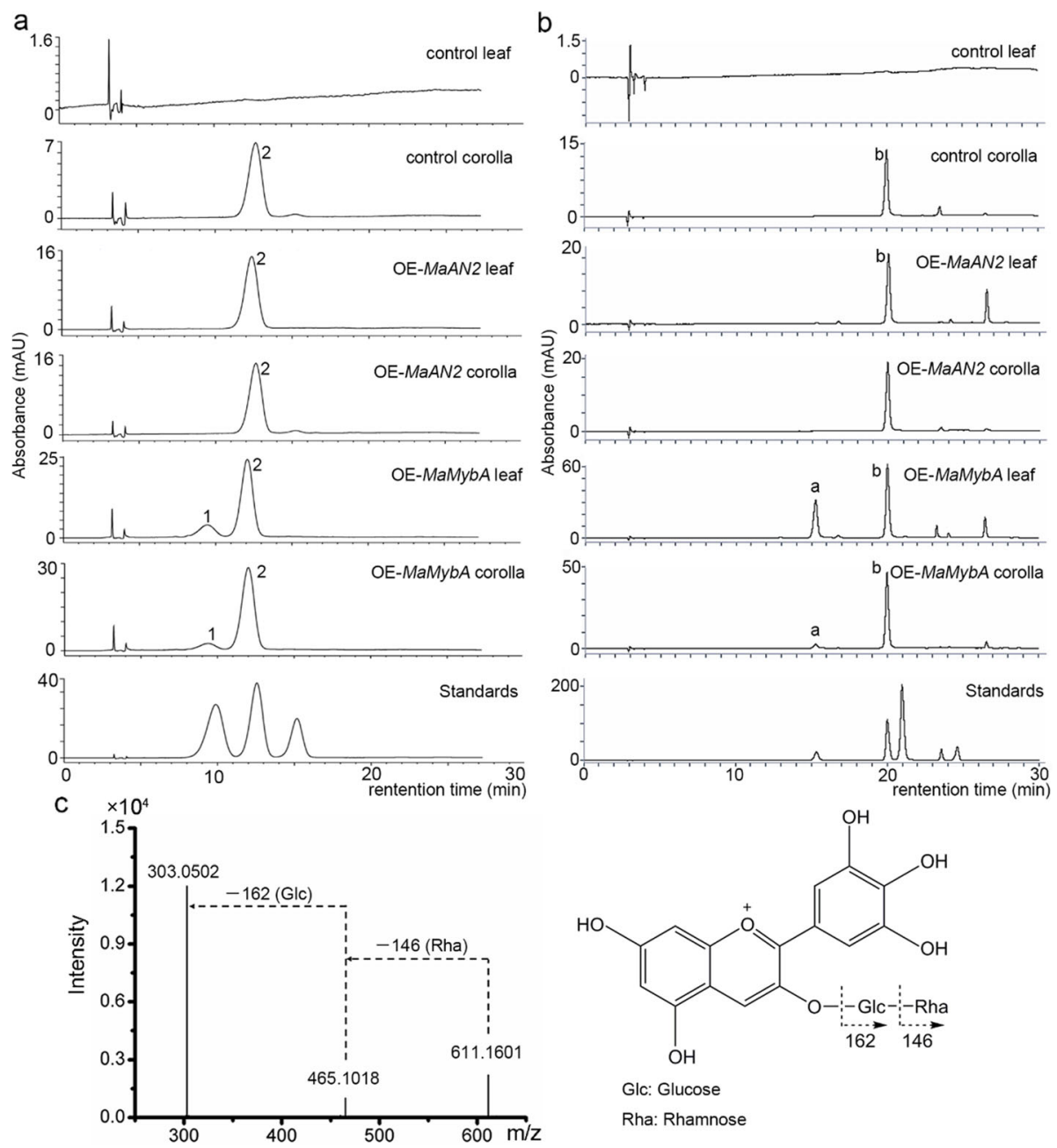

Fig. 5 Anthocyanin composition analysis of the leaves and corollas of the control, OE-MaAN2, and OE-MaMybA tobaccos. High-performance liquid chromatography analysis of anthocyanin extract (a), and its corresponding hydrolysate (b) in the leaves and corollas of the control, OEMaAN2, and OE-MaMybA tobaccos. According to the chromatographic peak in the sequence, the standards are delphinidin 3-rutinoside, cyanidin 3-rutinoside, and pelargonidin 3-rutinoside (from left to right) in diagram a; and delphinidin, cyanidin, petunidin, pelargonidin, and malvidin (from left to right) in diagram $\mathbf{b}$. The mass spectrum and structure patterns of delphinidin 3-rutinoside (c)

peaks 1 and 2 (Fig. 5a), respectively; and Dp (peak a) and Cy (peak b) were detected by hydrolysis (Fig. 5b). Therefore, we made a preliminary speculation that peaks 1 and 2 represented Dp3R and Cy3R, respectively. Moreover, the two anthocyanins were confirmed by ultra-performance liquid chromatography (UPLC)time-of-flight (TOF)-tandem mass spectrometry (MS/ MS) analysis. In detail, peak 1 showed typical molecular ions at a mass-to-charge ration $(\mathrm{m} / \mathrm{z})$ of 611.1601 $[\mathrm{M}]^{+}\left(\mathrm{C}_{27} \mathrm{H}_{31} \mathrm{O}_{16}\right)$, which was consistent with the molecular weight of $\mathrm{Dp} 3 \mathrm{R}$. In addition, two product ions, one at $\mathrm{m} / \mathrm{z} 465.1018$ and one at $\mathrm{m} / \mathrm{z} 303$, indicated that one molecule of glucose and one of rhamnose were released from one molecule of Dp (Fig. 5c). Additionally, the metabolite was also used to search the SciFinder and Reaxys databases. Peaks 1 and 2 were therefore identified as representing $\mathrm{Dp} 3 \mathrm{R}$ and Cy3R (Additional file 5: Figure S5), respectively.

Additionally, microscopic observations indicated that no anthocyanin was found in any of these cell types in the control tobacco (Additional file 6: Figure S6ad). Anthocyanin accumulated primarily in the palisade parenchyma, abaxial epidermal cells, and trichomes in OE-MaAN2 and OE-MaMybA tobacco leaves (Additional file 6: Figure S6e-l). Anthocyanin was also scattered in the adaxial epidermal cells, spongy parenchyma, and hypodermal parenchyma cells (Additional file 6: Fig. S6g, h, k, l). Moreover, there were no morphological changes in the epidermal cells of leaves and corollas between the control, OE-MaAN2, and $\mathrm{OE}-M a M y b A$ plants, according to scanning electron microscopy (Additional file 7: Figure S7). 
Overexpression of $M a M y b A$ in tobacco upregulated the expression levels of anthocyanin pathway genes

We first performed a quantitative real-time polymerase chain reaction (qRT-PCR) assay to test $M a M y b A$ transcripts in the transgenic tobaccos, and found that $M a M y b A$ was expressed in the leaves and corollas of three transgenic lines (OE\#1, OE\#2, and OE\#3), but not in the negative control plants (i.e., control, Fig. 6a, d). In addition, MaAN2 was also expressed in the positive control tobacco (i.e., OE-MaAN2, Fig. 6a, d). Next, we conducted a qRTPCR assay of anthocyanin biosynthetic and TF genes in the leaves and corollas of the control, OE-MaAN2, and OE$M a M y b A$ tobacco. The results showed that all the anthocyanin structural genes, including $\mathrm{NtCHS}, \mathrm{NtCHI}, \mathrm{NtF} 3 \mathrm{H}$, $N t F 3 ' H, N t F 3^{\prime} 5^{\prime} H, N t D F R, N t A N S$, and NtUFGT, were upregulated in the leaves and corollas of transgenic plants, especially in OE\#2 and OE\#3 (Fig. 6b, e). The two endogenous bHLH TF genes NtAn1a and NtAn1b, which regulate flavonoid synthesis in tobacco [32], were also upregulated in the leaves and corollas of the OE-MaMybA plants (Fig. 6c, f). However, the transcripts of NtAN2 were barely detected in the leaves of all genotypes of tobaccos, and those in the corollas of OE-MaAN2 and OE-MaMybA plants were lower than those in the control (Fig. 6c, f).

\section{Discussion}

Anthocyanins are the primary pigments in flowers. Anthocyanin biosynthesis is regulated by R2R3-MYB TFs, or the
MBW complex [33]. R2R3-MYB TFs involved in flower coloration have been found in various ornamental plants, including Gerbera hybrida, Lilium, and Anthurium [14-16]. In grape hyacinth, previous study has shown that an anthocyanin-related R2R3-MYB, MaAN2, induced the obvious increment of cyanidin biosynthesis in tobacco and relied on AtTT8 or MabHLH1 to activate the LBG expression [17] (Fig. 3b). In the present study, we isolated a novel R2R3-MYB, MaMybA, which differed from MaAN2. Heterologous expression of $M a M y b A$ conferred intense and dark anthocyanin pigmentation in tobacco, and produced a new anthocyanin, Dp3R. The study determined some precise differences between MaMybA and MaAN2 functioning in anthocyanin biosynthesis, and further revealed the regulatory mechanism responsible for coloration in $M$. armeniacum flowers.

\section{MaMybA is an R2R3-MYB TF in the AN2 subgroup of the R2R3-MYB family}

Anthocyanin-promoting R2R3-MYB TFs have specific structures and typical motifs. In $M$. armeniacum, we characterized an R2R3-MYB MaMybA, similar to MaAN2, which contained three conserved amino acids (Arg, Val, and Ala) in the highly conserved R2R3 domain of N-terminal region and a typical motif $[\mathrm{K} / \mathrm{R}] \mathrm{P}[\mathrm{Q} /$ $R] P[Q / R]$ in the $C$-terminal region. These conserved amino acids and typical motif have also been reported as

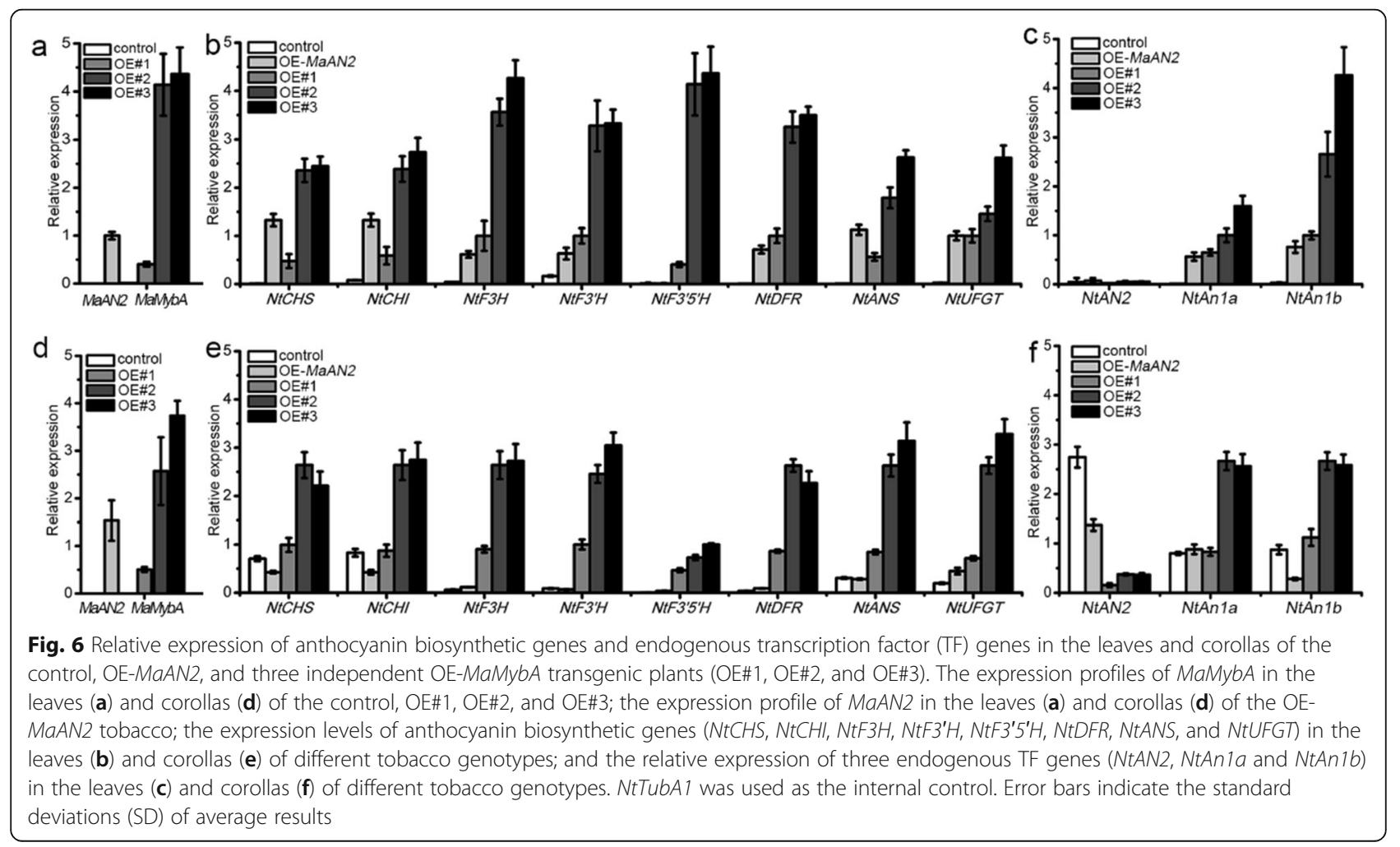


present in anthocyanin-promoting R2R3-MYBs of the AN2 subgroup in dicots [7, 11, 20] and a few monocots [15-17, 34] (Fig. 1a, b). Notably, MaMybA and MaAN2 in monocot plants were not found to contain the motif $\mathrm{KAX}[\mathrm{K} / \mathrm{R}] \mathrm{C}[\mathrm{S} / \mathrm{T}]$ in the $\mathrm{C} 1$ subgroup of R2R3MYBs from the Poaceae and Orchidaceae plant families (Fig. 1a, b). Additionally, the motif $[\mathrm{D} / \mathrm{E}] \mathrm{LX}_{2}[\mathrm{R} /$ $\mathrm{K}] \mathrm{X}_{3} \mathrm{LX}_{6} \mathrm{LX}_{3} \mathrm{R}$ in the $\mathrm{R} 3$ repeat of MaMybA and MaAN2 (Fig. 1a) indicated that MaMybA and MaAN2 could interact with bHLH TFs. MaMybA was identified as a TF because it was localized in the cell nucleus, and had transcriptional activation ability (Fig. 2, Additional file 1: Figure S1).

To our knowledge, all anthocyanin-related dicot R2R3-MYB TFs have been grouped into the AN2 subgroup of the R2R3-MYB family. In monocots, only those MYBs in Poaceae and Orchidaceae are clustered into the C1 subgroup [30, 35, 36]; the other monocot MYBs are placed in the AN2 subgroup [16, 17, 25, 26, 34]. Therefore, more anthocyanin-related R2R3-MYBs should be excavated in order to determine the regularity of clustering between dicots and monocots.

Interestingly, phylogenetic analysis indicated that the evolutionary relationship of MaMybA was closer than that of MaAN2 to AcMYB1. MaMybA and MaAN2 shared an identity of only $43.5 \%$ in their whole amino acid sequences. The relative expression of $M a M y b A$ in different tissues indicated that it was predominantly expressed in pigmented flowers (Fig. 1c). Its expression was coordinated with, although slightly earlier than that of MaAN2, and also with pigment accumulation during flower development [17].

The large family of R2R3-MYB is the major family of MYB proteins. R2R3-MYBs all have the highly conserved $\mathrm{R} 2 \mathrm{R} 3$ domain at the $\mathrm{N}$-termini, which decide the regulatory specificity and offer the MYB nuclear localization signals, while the C-terminal regions often possess transactivation or DNA-repression motifs [37, 38]. In Arabidopsis, the R2R3-MYBs are divided into 22 subgroups according to the recurring amino acid motifs in the C-termini [39]. Specifically, the R2R3-MYBs of AN2 subgroup (i.e., subgroup 6) which consist of the conserved motif $([K / R] P[Q / R] P[Q / R])$ in the C-terminal regions function in inducing anthocyanin biosynthesis [34]; the R2R3-MYBs of subgroup 4 contained the conserved EAR motif (LxLxL) in the C-termini function in reducing anthocyanin biosynthesis [40]. Previous study also showed that R2R3-MYBs which contain highly conserved motifs and exon lengths have similar functions between Arabidopsis and Vitis, although low sequence homology in the $\mathrm{C}$-terminal regions of all R2R3-MYB proteins. Thus, despite occurrence of diversification, the highly conserved regions could maintain the specific functionality, while variations in
C-terminal regions could facilitate the gaining of new or cooperative functions [37].

In planta, there are numerous examples where two or more R2R3-MYB genes, functioning in anthocyanin biosynthesis, are found within a single plant species, such as AtPAP1 and AtPAP2 in Arabidopsis [20]. However, some R2R3-MYB anthocyanin regulators found in the same plant show diversity through functioning in anthocyanin pigmentation in different tissues or organs; for example, Petunia AN2 is responsible for flower limb pigmentation, while AN4 mainly regulates anther anthocyanin accumulation [41]; in Solanum lycopersicum, two different R2R3 MYB, SIANT1 and SIAN2, both could induce anthocyanin biosynthesis, but only the latter one functions in anthocyanin synthesis in vegetative tissues under high light or low temperature conditions [42]. In grapevine, four anthocyanin-related R2R3 MYBs have different amino acid lengths of C-termini. Particularly, VvMYBA2 has two C-terminal repeats, while VvMYBA1 has one and VvMYBA3 lacks this motif. Besides, MYBA1 and MYBA2 function fruit coloration, but MYBA3 loses the function of accumulating anthocyanin. These differences could cause novel or cooperative functions [43]. In this study, each of MaMybA and MaAN2 only contained one conserved motif (KPQPQ) in the Cterminal region. Furthermore, premature termination of the C-terminal domain did not occur in MaMybA or MaAN2 (the amino acid lengths of MaMybA and MaAN2 were 237 and 240, respectively), and the two MYBs could perform complete functions in anthocyanin biosynthesis in tobacco. Thus, MaAN2 might be the paralog of MaMybA by gene duplication in the process of evolution, and the variation in C-terminal indicated that they might possess redundant, overlapping or cooperative functions in grape hyacinth. So, based on this, the experimental evidence on functionality of the variable C-terminal between MaMybA and MaAN2 is a future direction of our work.

\section{MaMybA alone or in interaction with MabHLH1 could activate anthocyanin biosynthetic gene promoters}

Anthocyanin biosynthesis is regulated by MYB-bHLH complexes in almost all plant species [33]. In the present study, the resulting MabHLH1 contained an MYB interaction region on the $\mathrm{N}$-terminal and a bHLH DNA binding domain at the $\mathrm{C}$-terminal region, together with other IIIf bHLH proteins involved in flavonoid biosynthesis [28, 30, 44] (Additional file 2: Figure S2a). The phylogenetic analysis indicated that MabHLH1 was grouped into the LC/JAF13/DEL clade of the IIIf bHLH TF family [26, 45] (Additional file 2: Figure S2b). These findings indicated that MabHLH1 might play a role in anthocyanin biosynthesis in $M$. armeniacum. However, MabHLH1 was constitutively expressed in different 
tissues, and was not concomitant with the pigmentation of flower development (Additional file 2: Figure S2c), which has also been found in apples [46], grapes [47], and Phalaenopsis [36].

MabHLH1 interacted with either MaMybA or MaAN2 in vivo; this was confirmed by the BiFC assay (Fig. 3a). In the dual luciferase assay, we found that the promoter activity of MaCHS (EBG) was induced by none of all TFs (each or both of MYB-bHLH complexes) in this assay, which was consistent with the result that the activity of $\mathrm{MrCHS}$ promoter was not response to MrMYB1, or MrbHLH1, or MrMYB1-MrbHLH1 in Myrica rubra [48]. MaMybA alone was able to regulate the expression of MaDFR (LBG), in contrast to MaAN2, which relied on MabHLH1 to control MaDFR expression (Fig. 3b). In maize, $\mathrm{ZmP}$ alone can activate flavonoid biosynthetic genes, but $\mathrm{ZmC1}$ require a direct interaction with bHLH protein R/B to do so [49]; in Phalaenopsis, PeMYB11 is dependent on PebHLH1, while PeMYB2 and PeMYB12 are independent of PebHLH1 in regulating PeDFR expression [36]. The regulation pattern of $\mathrm{ZmP}, \mathrm{PeMYB2}$, and PeMYB12 is consistent with that of MaMybA. However, in Arabidopsis, AtPAP1 interacts with AtTT8 or AtGL3 to regulate AtDFR expression [50, 51]. PhAN2 also requires bHLH cofactor PhAN1 or PhJAF13 to enhance promoter activity in Petunia PhDFR [44]; and the MdMYB10MdbHLH3 and MdMYB10-MdbHLH33 complexes can each activate the expression of MdDFR in apples [28]. These regulation patterns are highly consistent with that of MaAN2.

\section{Phenotypic differences between OE-MaMybA and OE-MaAN2 tobacco can be ascribed to the high concentration of anthocyanin and the newly produced Dp3R in OE-MaMybA tobacco}

The ectopic expression of a gene in a model plant quickly facilitates its functional identification, and visible anthocyanin pigmentation could directly demonstrate its function in anthocyanin biosynthesis [52]. Many studies show that overexpressing anthocyanin-related R2R3MYB genes could promote anthocyanin production in tobacco by genes such as AtPAP1 [18, 20], and AaMYB2 [16]. In this study, overexpressing $M a M y b A$ in tobacco induced a high level of anthocyanin production in the leaf, petal, anther, and calyx, which all presented with mulberry pigmentation (Fig. 4a-c), while the ovary wall and seed skin were barely pigmented (Fig. 4c, d). However, OE-MaAN2 tobacco presented a magenta leaf, calyx, anther, and a deep pink corolla, which was much lighter than in OE-MaMybA tobacco (Fig. 4a-c); the ovary wall and seed skin of OE-MaAN2 tobacco showed a reddish color, which were different from that of OE$M a M y b A$ (Fig. 4c, d). A microscopic observation of OE-
$M a M y b A$ tobacco leaves indicated that anthocyanin was primarily present in the palisade parenchyma, trichomes, and lower epidermis, with some pigment scattered in the upper epidermis and spongy mesophyll; and the pigmentation was much deeper in comparison with OE-MaAN2 transgenic plants (Additional file 6: Figure S6). In OEAtPAP1 tobacco, the pigmentation cells were primarily found in the trichomes and lower epidermis [18]. Thus, different R2R3-MYBs influence anthocyanin accumulation in different tissues or cells to various extents, and the distribution of anthocyanin might affect plant color.

Previous studies have demonstrated that the primary anthocyanin in the tobacco corolla is Cy3R [31]. In the present study, a newly produced anthocyanin, Dp3R, was found in the leaves and corollas of OE-MaMybA tobacco, in addition to Cy3R, the primary anthocyanin (Fig. 5a); this differed from OE-MaAN2 tobacco, in which the primary anthocyanin in the leaves and corollas was found to be Cy3R (Fig. 5a). Additionally, the anthocyanin levels of the leaves and corollas in three OE$M a M y b A$ transgenic lines were all greater than $2.0 \mathrm{mg}$ $\mathrm{g}^{-1}$ fresh weight (FW), while those in OE-MaAN2 plants were both lower $2.0 \mathrm{mg} / \mathrm{L} \mathrm{FW}$ (Fig. 4e, f). Previous study has shown that two anthocyanin regulatory genes (Arabidopsis R2R3 MYB gene mPAP1 and Zea mays bHLH-type gene B-Peru) overexpressing in N. tabacum L. to yield an enhanced anthocyanin content, and also produced a new type pigment Dp3R in the flower limb of transgenic tobacco, which presented a dark red color [53]. The hydrolysate of anthocyanin extracts from the leaves and corollas of OE-MaMybA tobacco also showed that $\mathrm{Cy}$ was the primary anthocyanidin, followed by Dp (Fig. 5b); this was similar to the results of anthocyanin hydrolysis from OE-AtPAP1 tobacco [18]. Previous studies have shown that the reddish-purple flower of grape hyacinth ( $M$. comosum 'Plumosum') contains two main anthocyanidins (Dp and $\mathrm{Cy}$ ) that contribute to its color [22]. Therefore, we concluded that the red Cy-based derivatives, together with the blue Dp-based derivatives and the high anthocyanin concentration, contributed to the dark reddish-purple color in OE-MaMybA tobacco. Moreover, when overexpressing different R2R3-MYB genes in model plants, the resultant plant phenotypes were distinct in anthocyanin localization, type and content, possibly because different R2R3-MYB TFs regulate different target genes to induce different anthocyanins in different tissues.

R2R3-MYB proteins of MBW complexes play core roles in anthocyanin accumulation, and the MBW complexes could probably induce the entire anthocyanin biosynthesis pathway [8]. In the present study, expression levels of almost all the anthocyanin biosynthetic genes (NtCHS, NtCHI, NtF3H, NtF3'H, NNtDFR, NtANS, and NtUFGT) and endogenous bHLH TF genes (NtAnla 
and $N t A n 1 b)$ were upregulated in the leaves and corollas of three OE-MaMybA transgenic lines (especially in $\mathrm{OE \# 2}$ and $\mathrm{OE \# 3;} \mathrm{Fig.} \mathrm{6);} \mathrm{this} \mathrm{was} \mathrm{consistent} \mathrm{with} \mathrm{the} \mathrm{re-}$ sults for the leaves of OE-AaMYB2 and OE-MaAN2 tobacco [16, 17] (Fig. 6b, c). However, the transcripts of $N t A N 2$ were not detected in the leaves of all detected tobaccos (Fig. 6c), because NtAN2 is a floral tissue-specific anthocyanin-related R2R3-MYB in N. tabacum [54]. Moreover, the expression levels of NtAN2 in the corollas of transgenic plants were lower than that in control (Fig. 6f); this was similar to the results of the overexpressing B-peru and $m P A P 1$ in tobacco, which indicated that excess anthocyanin pigmentation could inhibit the expression of endogenous gene by feedback regulation [53]. Notably, NtF3' $5^{\prime} \mathrm{H}$ transcripts in the leaves and corollas of OE-MaMybA tobacco were much higher than in the control and OEMaAN2 plants (Fig. 6b, e). These results indicated that MaMybA promoted anthocyanin accumulation by upregulating the anthocyanin biosynthetic genes in the synthesis pathway. These upregulated anthocyanin biosynthetic genes led to an increase in metabolites, which formed the substrates for the subsequent catalytic reaction and therefore enabled the accumulation of a high concentration of anthocyanins. Additionally, the highly expressed $N t F 3^{\prime} 5^{\prime} H$ was responsible for catalyzing the product of $\mathrm{Dp} 3 \mathrm{R}$ that was also found in the flower limbs of transgenic tobacco in previous study [53].

\section{Conclusions}

In this study, a novel R2R3-MYB TF, MaMybA, which was identified from the flower transcriptome of $M$. armeniacum, was found to play a role in anthocyanin pigmentation. We determined the function of MaMybA in anthocyanin biosynthesis by heterologous expression in tobacco, and that the intense and dark reddish-purple anthocyanin pigmentation of OE-MaMybA tobacco could be attributed to the high concentration of anthocyanin and the newly produced Dp3R. The results indicated that $M a M y b A$ might be a candidate gene for modifying flower color in ornamental plants and potentially in engineering high levels of anthocyanins. Moreover, we also determined the similarities and differences in anthocyanin synthesis between MaMybA and MaAN2: they both could interact with a bHLH protein, but the former was independent whilst the latter was dependent of the bHLH to regulate LBGs. Besides, MaMybA was different from MaAN2 in inducing the content and composition of anthocyanin and coloring tissues in tobacco, which suggested that future work focuses on their functions in grape hyacinth.

\section{Methods}

\section{Plant materials}

Grape hyacinth ( $M$. armeniacum) plants were grown in an experimental field at Northwest A\&F University in
Yangling District, Shaanxi province, China. The tepals were divided into five stages (S1 S5) mainly according to the degree of petal pigmentation: $\mathrm{S} 1$ indicated no pigmentation; S2 showed pigmentation visible on the basal part; S3 indicated pigment beginning to turn blue; S4 presented that the flowers were completely blue, but had not opened; and S5 indicated that flowers completely opened, according to the method of Chen et al. [17], and were then collected and stored, together with the vegetative tissues (roots, bulbs, and leaves), at $-80^{\circ} \mathrm{C}$ until use. Aseptic tobacco ( $N$. tabacum 'NC89') seedlings were used for genetic transformation at the four-leaf stage. The $\mathrm{T}_{1}$ generation transgenic tobacco plants were transferred from an aseptic culture room to a greenhouse. The mature, fully expanded tobacco leaves and fully opened flower limbs were picked and stored at $-80^{\circ} \mathrm{C}$ in readiness for the next test. Arabidopsis thaliana (Col-0) and $N$. benthamiana plants were grown in a light incubator under a $16 \mathrm{~h}$ light $/ 8 \mathrm{~h}$ dark cycle until the four to six-leaf stage was achieved for cloning the AtDFR promoter and AtHY5 gene, and for dual luciferase assay, respectively.

\section{Quantitative real-time PCR assay}

Total RNA extraction from different tissues of $M$. armeniacum and tobacco, cDNA synthesis, and a qRT-PCR assay were undertaken using the protocols described by Chen et al. [17]. The qRT-PCR primers are listed in Additional file 8: Table S1. MaActin and NtTubA1 were used as the internal control genes for $M$. armeniacum and tobacco, respectively. Analysis was performed using three samples, with three replicates.

\section{Gene cloning}

Local BLASTP software was used to screen one anthocyanin-related R2R3-MYB and one anthocyaninrelated bHLH unigene, using AtPAP1 and AtTT8 from a transcriptome of $M$. armeniacum flowers [23]; the unigenes were designated $M a M y b A$ and MabHLH1, respectively. However, the R2R3-MYB unigene lacked the $5^{\prime}$ - and $3^{\prime}$-untranslated regions. Thus, we used the $5^{\prime}$-RACE and $3^{\prime}$-RACE methods to obtain the full-length cDNA sequence of $M a M y b A$ from $M$. armeniacum flowers. A detailed protocol for obtaining the full-length cDNA of MaMybA was followed in accordance with the methods described by Chen et al. [17] and Huang et al. [55]. The fulllength cDNA of MabHLH1 was cloned from $M$. armeniacum flowers using RT-PCR; the primers used for gene cloning are listed in Additional file 8: Table S1. The resulting cDNA sequences were submitted to the NCBI GenBank database under the accession numbers MF663728 (MaMybA) and MF663729 (MabHLH1). 
Sequence alignment and phylogenetic analysis

The deduced amino acid sequences of $M a M y b A$ and MabHLH1, as well as the other anthocyanin-related R2R3-MYBs and bHLH proteins retrieved from the GenBank database, were used for sequence alignment and phylogenetic analysis, respectively. The multiple sequence alignment was analyzed using DNAMAN software version 8.0 (Lynnon Biosoft, CA, USA). The phylogenetic tree was first analyzed using CLUSTALW software and then constructed with MEGA software version 6.0 [56], using the maximum-likelihood method. The bootstrap was set at 1000 replicates. The accession numbers of the different protein types are listed in Fig. 1 and Additional file 2: Figure S2, respectively.

\section{Subcellular localization and transcription activation ability assay}

For the subcellular localization assay, the ORF of either $M a M y b A$ or $M a b H L H 1$, without a termination codon, was inserted into pCambia2300 (pC2300)-GFP using a Seamless Cloning and Assembly Kit (Novoprotein, Shanghai, China) to generate either pC2300-MaMybAGFP (MaMybA-GFP) or pC2300-MabHLH1-GFP (MabHLH1-GFP), respectively. Additionally, the fulllength cDNA sequence of AtHY5 (AT5G11260), which encodes a nuclear localization marker protein, was cloned from the leaves of $A$. thaliana (Col-0) and inserted into pBI221-mCherry to generate pBI221AtHY5-mCherry (AtHY5-mCherry). The primers are listed in Additional file 8: Table S1. pC2300-GFP (35S: GFP) was used as a positive control. The various plasmids were cotransformed into $A$. thaliana mesophyll protoplasts using the protocol described by Yoo et al. [57]. The transformed protoplasts were cultivated for 16 $\mathrm{h}$ and then visualized using a laser scanning confocal microscope (Leica TCS SP8, Wetzlar, Germany).

In order to construct yeast expression vectors for measuring the transcription activation ability of MaMybA and MabHLH1, the two ORF PCR products were separately fused into pGBKT7. The primers are listed in Additional file 8: Table S1. pGBKT7-53 was cotransformed with pGADT7-T as a positive control, and pGADT7 was used as a negative control. The yeast transformation and autoactivation testing were each performed as described in Chen et al. [17].

\section{Bimolecular fluorescence complementation assay}

In order to verify the interaction between bHLH protein and R2R3-MYBs from M. armeniacum, a BiFC assay was conducted on the leaves of $N$. benthamiana to confirm the interaction between MabHLH1 and either MaMybA or MaAN2 (anthocyanin-related R2R3-MYB in $M$. armeniacum; KY781168) [17]. The ORF sequences of MabHLH1 and MaAN2 were individually inserted into pSPYNE (R) $173(\mathrm{YN})$ to generate YN/MabHLH1 and $\mathrm{YN} / \mathrm{MaAN} 2$, respectively. Additionally, the ORF sequence without either the MaMybA or MabHLH1 termination codon was inserted into pSPYCE (M) (YC) to generate $\mathrm{YC} / \mathrm{MaMybA}$ and $\mathrm{YC} / \mathrm{MabHLH} 1$, respectively. The primers are listed in Additional file 8: Table S1. The recombinant and empty vector plasmids were transformed into GV3101 by electroporation, and the various transformed Agrobacterium cultures were co-infiltrated into $N$. benthamiana leaves. After being cultivated in a light incubator for $3 \mathrm{~d}$, the infiltrated tobacco leaves were observed and photographed using a laser scanning confocal microscope (Leica TCS SP8, Wetzlar, Germany).

\section{Dual luciferase assay}

For the dual luciferase assay, the full-length cDNA sequences of MaMybA, MaAN2, and MabHLH1 were each cloned into pGreenII 62-SK. The promoters of the EBG in $M$. armeniacum (MaCHS, KY781171) and the key LBGs in $M$. armeniacum (MaDFR, KY781169) and $A$. thaliana (AtDFR, AT5G42800) were each inserted into pGreenII 0800-LUC. The primers are listed in Additional file 8: Table S1. All the recombinant plasmids were electroporated into GV3101. Infiltration, transient expression analysis, and measurements of enzyme activity for LUC and Renilla luciferase (REN) were performed according to previously cited protocols $[17,58,59]$. The statistical analysis of the dual luciferase assay was conducted using four biological replicates.

\section{Stable tobacco transformation}

In order to verify the function of $M a M y b A$, the ORF sequence without termination codons for $M a M y b A$ was inserted into pCambia1304. The primers were listed in Additional file 8: Table S1. Thus, $M a M y b A$ was promoted by the $35 \mathrm{~S}$ promoter. The recombinant or empty vector plasmid was introduced into GV3101 by electroporation. The tobacco leaf disk transformation was performed according to the protocol described by Horsch et al. [60]. The transformed tobacco plants were screened using $25 \mathrm{mg} \mathrm{L}^{-1}$ hygromycin antibiotic, which was used as the plant selective marker. The next stage of analysis used $\mathrm{T}_{1}$ generation transgenic plants of overexpressing $M a M y b A$ (OE-MaMybA) that showed obvious color changes in the leaves and flowers. The method of obtaining the $\mathrm{T}_{1}$ generation transgenic tobaccos of overexpressing MaAN2 was followed according to the protocols of our previous study [17].

\section{HPLC analysis}

The method for extracting anthocyanin from the fresh, mature, fully expanded tobacco leaves and fully opened flower limbs of different transgenic tobacco genotypes was conducted as previously described by Chen et al. 
[17]. In addition, in order to obtain anthocyanin hydrolysate, a $300 \mu \mathrm{L}$ aliquot with an equal volume of $6 \mathrm{M} \mathrm{HCl}$ was boiled for $1 \mathrm{~h}$ to release anthocyanidin aglycones. All the extracts were filtered through a $0.22 \mu \mathrm{m}$ filter membrane and then subjected to reverse HPLC analysis. The HPLC experimental conditions, instruments, and protocols were performed according to Chen et al. [17]. The anthocyanin extracts were first identified with reference to commercial standards (all the standards mentioned are listed in Fig. 5; Sigma, USA). The anthocyanin content was determined according to the standard curve of Cy3R. The anthocyanin content in each tobacco sample was analyzed using three biological replicates.

\section{UPLC-triple-TOF-MS/MS conditions}

UPLC experiments were performed on an Acquity UPLC system (Waters, MA, USA) coupled with a C18 column $(1.8 \mu \mathrm{m}, 100 \mathrm{~mm} \times 4.6 \mathrm{~mm}$; Agilent ZORBAX$\mathrm{SB}, \mathrm{CA}, \mathrm{USA})$. The column temperature was maintained at $30^{\circ} \mathrm{C}$; the detection wavelength was $530 \mathrm{~nm}$. The eluent consisted of aqueous solution A $(0.1 \%$ formic acid in water) and organic solvent B $(0.1 \%$ formic acid in acetonitrile) at a flow rate of $0.8 \mathrm{~mL} \mathrm{~min}{ }^{-1}$. The gradient elution programme was conducted as follows: $0 \sim 2 \mathrm{~min}$, 5\% B; $25 \mathrm{~min}, 50 \%$ B; $35 \mathrm{~min}, 95 \%$ B; $37 \mathrm{~min}, 95 \%$ B; and $38 \mathrm{~min}, 5 \% \mathrm{~B}$. The injection volume was $5 \mu \mathrm{L}$.

Mass spectrometry (MS) was performed on a TOF mass spectrometer (Triple TOF $^{\text {ma }} 5600+$ system) with a DuoSpray $^{\mathrm{Tn}}$ source operating in the positive and negative electrospray ionization modes (AB SCIEX, CA, USA). The optimized operating parameters of the MS/MS detector were as follows: ion spray voltage, $-4.5 \mathrm{kV}$ (negative) and $5.5 \mathrm{kV}$ (positive); ion source heater, $550^{\circ} \mathrm{C}$ (negative) and $600^{\circ} \mathrm{C}$ (positive); curtain gas, ion source gas 1 , and ion source gas 2 set to 35,50 , and 50 psi, respectively; and the experiments were run using a $100 \sim 1000 \mathrm{~m} / \mathrm{z}$ scan. For the MS experiments, the declustering potential and collision energy levels were set at $100 \mathrm{~V}$ and $10 \mathrm{~V}$, respectively; the MS/MS data were collected using the TOF MS-Product Ion-IDA (information-dependent acquisition) mode; and the collision-induced dissociation energy levels were set to $-20,-40$, and $-60 \mathrm{~V}$. In addition, an automated calibration delivery system was used to simultaneously calibrate the MS and MS/MS experiments, in order to reduce the mass axis error to less than $2 \times 10^{-6}$.

\section{Histological localization of anthocyanins and scanning electron microscopy}

The epidermis was peeled from the leaf using fine-tipped forceps. Cross-sections of fresh leaf tissues were made by hand using a razor blade. The samples were immediately transferred to glass slides with a drop of water and observed under a microscope (Nikon Eclipse 50i, Tokyo, Japan).
The scanning electron microscopy was performed according to the previously published protocol described by Qi et al. [61]. Specifically, the pigmented leaves and corollas of transgenic tobacco were first fixed in $4 \%$ glutaraldehyde, and then dehydrated in an alcohol series. Next, the samples were critical point-dried and sputtercoated with platinum. Lastly, all the samples were observed under a scanning electron microscope (JEOL JSM-6360LV, Tokyo, Japan).

\section{Statistical analysis}

The statistical analysis was conducted using SPSS 20.0 software (SPSS Inc., Chicago, USA). Data are presented as means \pm standard deviations (SD). The levels of statistical significance were determined by Least Significant Difference $(L S D)$ analysis: $P<0.05$.

\section{Additional files}

Additional file 1: Figure S1. Transcription activation ability of MaMybA. Yeasts transformed with the pGBKT7/MaMybA vector (positive control (pGBKT7-53 + pGADT7-T) and negative control (pGBKT7)), were each cultured in SD/-Trp media, SD/-Trp media with $40 \mathrm{mg} \mathrm{mL}^{-1} \mathrm{X}$-a-gal, and $\mathrm{SD} /$-Trp media added to $40 \mathrm{mg} \mathrm{mL}^{-1} \mathrm{X}$-a-gal and $200 \mathrm{ng} \mathrm{mL}^{-1} \mathrm{AbA}$. (DOCX $273 \mathrm{~kb}$ )

Additional file 2: Figure S2. Alignment and phylogenetic analysis of the deduced amino acid sequence of MabHLH1 and other basic HelixLoop-Helix (bHLH) proteins associated with flavonoid biosynthesis from other plants. a Sequence alignment of partial amino acids deduced from MabHLH1 and other bHLH proteins from other plant species. The MYB-interaction and bHLH DNA binding regions are indicated above the alignment by bold black lines. $\mathbf{b}$ Phylogenetic analysis of entire amino acid sequences deduced from MabHLH1 and other bHLH proteins from other plants. The maximum-likelihood phylogenetic tree was generated using MEGA 6.0 software. Numbers next to the nodes indicate the bootstrap values from 1000 replications. The bHLH protein sequences of different plant species were retrieved from GenBank database and their GenBank accession numbers are as followings: Arabidopsis thaliana: AtTT8 (NP_1927202), AtEGL3 (NP_0011853021), AtGL3 (NP_0013327061), and AtMYC1 (AEE818881); Petunia hybrida: PhJAF13 (AAC394551) and PhAN1 (AAG259281); Nicotiana tabacum: NtAN1a (AEE992571) and NtAN1b (AEE992581); Antirrhinum majus: AmDELILA (AAA326631); Gerbera hybrida: GMYC1 (CAA076151); Vitis vinifera: VVMYC1 (ACC686851) and VVMYCA1 (ABM923323): Malus domestica: MdbHLH3 (ADL365971) and MdbHLH33 (ABB844741); Myrica rubra: MrbHLH1 (AGO583721); Perilla frutescens: PfMYC-RP (BAA75513); Brassica rapa: BrTT8 (AEA032811); Medicago truncatula: MtTT8 (AKN796061); Lotus japonicus: LjTT8 (BAH288811); Dahlia pinnata: DvIVS (BAJ335151); Ipomoea nil: InbHLH1 (BAE943931) and InbHLH2 (BAE943941); Fragariaxananassa: FabHLH3 (AFL024631) and FabHLH33 (AFL024651); Prunus persica: PpbHLH3 (AlE575081); Prunus avium: PabHLH3 (AJB284811) and PabHLH33 (AJB284841); Litchi chinensis: LcbHLH3 (APP941241), LcbHLH1 (APP941221), and LcbHLH2 (APP941231); Lilium hybrid: LhbHLH1 (BAE200571) and LhbHLH2 (BAE200581); Zea mays: ZmLC (NP_0011053391); Dendrobium hybrid: DhbHLH1 (AQS798531): Gentiana triflora: GtbHLH1 (BAH033871). c Expression profile of MabHLH1 in different tissues of Muscari armeniacum. MaActin was the reference gene. Error bars indicate the standard deviations (SD) of average results. (DOCX $1686 \mathrm{~kb}$ )

Additional file 3: Figure S3. Subcellular localization and transcription activation ability of MabHLH1. a Subcellular localization of MabHLH1. The transient co-expression of MabHLH1-GFP with AtHY5-mCherry in Arabidopsis thaliana mesophyll protoplasts showed that MabHLH1 and AtHY5 were co-localized in the nucleus. Bars: $10 \mu \mathrm{m}$. b Transcription activation ability of MabHLH1. Yeasts transformed with the positive 
control (pGBKT7-53 + pGADT7-T) and negative control (pGBKT7), and pGBKT7/ MabHLH1 vectors were each cultivated in SD/-Trp media, SD/Trp media with $40 \mathrm{mg} \mathrm{mL}^{-1} \mathrm{X}$-a-gal, and SD/-Trp media plus $40 \mathrm{mg} \mathrm{mL}^{-}$ ${ }^{1} \mathrm{X}$-a-gal and $200 \mathrm{ng} \mathrm{mL}^{-1}$ AbA. The positive control and pGBKT7/ MabHLH1 exhibited blue yeast plaques, while the negative control did not grow in SD/-Trp media plus $40 \mathrm{mg} \mathrm{mL}^{-1}$ X-a-gal and $200 \mathrm{ng} / \mathrm{mL}$ AbA. (DOCX $280 \mathrm{~kb})$

Additional file 4: Figure S4. Anthocyanin composition analysis of the leaves and corollas in the other two lines of control, OE-MaAN2, and OE-MaMybA tobaccos. High-performance liquid chromatography analysis of anthocyanin extract. According to the chromatographic peak in the sequence, the standards are delphinidin 3-rutinoside, cyanidin 3-rutinoside, and pelargonidin 3-rutinoside (from left to right). (DOCX $228 \mathrm{~kb}$ )

Additional file 5: Figure S5. Mass spectrum and structure patterns of cyanidin-3-rutinoside. (DOCX $153 \mathrm{~kb}$ )

Additional file 6: Figure S6. Histological localization of anthocyanins in leaf tissues of the control, OE-MaAN2, and OE-MaMybA tobaccos. The genotypes are the control (a-d), OE-MaAN2 (e-h), and OE-MaMybA (i-l); abaxial epidermis (a, e, and $\mathbf{i})$, trichomes $(\mathbf{b}, \mathbf{f}$, and $\mathbf{j})$, cross-sections through leaves ( $\mathbf{c}, \mathbf{g}$, and $\mathbf{k}$ ), and leaf veins ( $\mathbf{d}, \mathbf{h}$, and $\mathbf{I})$. ep, epidermal; hy, hypodermal; tr, trichome; pa, parenchyma; pp., palisade parenchyma; st, stomata; sp., spongy parenchyma; va, vascular bundle. Bars: $100 \mu \mathrm{m}$. (DOCX $550 \mathrm{~kb}$ )

Additional file 7: Figure S7. Scanning electron microscopy images of leaf and corolla adaxial surface cells in different tobacco genotypes. The leaf (a-c) and corolla ( $\mathbf{d}-\mathbf{f})$ adaxial surface cells are shown for the control (a, d), OE-MaAN2 (b, e), and OE-MaMybA (c, f) tobacco. (DOCX 145 kb)

Additional file 8: Table S1. Primers used in this study. (DOCX $22 \mathrm{~kb}$ )

\section{Abbreviations}

AbA: Aureobasidin A; ANS: Anthocyanidin synthase; bHLH: Basic helix-loophelix; BiFC: Bimolecular fluorescence complementation; $\mathrm{CHI}$ : Chalcone isomerase; CHS: Chalcone synthase; Cy: Cyanidin; Cy3R: Cyanidin 3-rutinoside; DFR: Dihydroflavonol 4-reductase; Dp: Delphinidin; Dp3R: Delphinidin 3rutinoside; EBG: Early biosynthetic gene; F3'H: Flavonoid 3'-hydroxylase; F3' $5^{\prime}$ H: Flavonoid 3'5'-hydroxylase; F3H: Flavanone 3-hydroxylase; GFP: Green fluorescent protein; HPLC: High-performance liquid chromatography; LBG: Late biosynthetic gene; LUC: Firefly luciferase; m/z: Mass-to-charge ratio; MS: Mass spectrometry; MS/MS: tandem mass spectrometry; OE: Overexpressing; ORF: Open reading frame; GRT-PCR: Quantitative realtime polymerase chain reaction; REN: Renilla luciferase; RT-PCR: Reverse transcription-polymerase chain reaction; TF: Transcription factor; TOF: Timeof-flight; UFGT: Uridine diphosphate-sugar: flavonoid glycosyltransferases; UPLC: Ultra-performance liquid chromatography; WDR: WD-repeat; YFP: Yellow fluorescent protein

\section{Acknowledgements}

We thank Wenkong Yao for his kind support in conducting the microscopy analysis and also thank Elsevier's WebShop Editing Service for language editing.

\section{Authors' contributions}

YL conceived the study. KC and LD conducted the experiments. HL participated in parts of the experiments. $\mathrm{KC}$ analyzed the data and wrote the manuscript. All the authors read and approved the manuscript.

\section{Funding}

This work was supported by the National Natural Science Foundation of China (grant number 31471905), the National Natural Science Foundation of China (grant number 31170652), and the Natural Science Basic Research Plan in the Shaanxi Province of China (grant number 2017JQ3019). The funding bodies had no role in the design, collection, and analysis, interpretation of data or in writing the manuscript.

\section{Availability of data and materials}

All data generated or analyzed during this study are included in this published article and its supplementary information files.
Ethics approval and consent to participate

Not applicable.

\section{Consent for publication}

Not applicable.

\section{Competing interests}

The authors declare that they have no competing interests.

\section{Author details}

${ }^{1}$ College of Landscape Architecture and Arts, Northwest A\&F University, Yangling 712100, Shaanxi, People's Republic of China. ${ }^{2}$ Key Laboratory of Horticultural Plant Biology and Germplasm Innovation in Northwest China, Ministry of Agriculture, Yangling 712100, Shaanxi, People's Republic of China. ${ }^{3}$ State Key Laboratory of Crop Stress Biology in Arid Areas, Northwest A\&F University, Yangling 712100, Shaanxi, People's Republic of China.

\section{Received: 25 February 2018 Accepted: 29 August 2019}

Published online: 09 September 2019

\section{References}

1. Zhao D, Tao J. Recent advances on the development and regulation of flower color in ornamental plants. Front Plant Sci. 2015;6:261.

2. Polturak G, Grossman N, Velacorcia D, Dong Y, Nudel A, Pliner M, et al. Engineered gray mold resistance, antioxidant capacity, and pigmentation in betalain-producing crops and ornamentals. Proc Natl Acad Sci U S A. 2017;114:9062-7.

3. Tanaka Y, Brugliera F, Chandler S. Recent progress of flower colour modification by biotechnology. Int J Mol Sci. 2009;10:5350-69.

4. Katsumoto Y, Fukuchi-Mizutani M, Fukui Y, Brugliera F, Holton TA, Karan M, et al. Engineering of the rose flavonoid biosynthetic pathway successfully generated blue-hued flowers accumulating delphinidin. Plant Cell Physiol. 2007:48:1589-600

5. Azadi P, Bagheri H, Nalousi AM, Nazari F, Chandler SF. Current status and biotechnological advances in genetic engineering of ornamental plants. Biotechnol Adv. 2016:34:1073-90

6. Nishihara M, Nakatsuka T. Genetic engineering of novel flower colors in floricultural plants: recent advances via transgenic approaches. Methods $\mathrm{Mol}$ Biol. 2010;589:325

7. Elomaa P, Uimari A, Mehto M, Albert VA, Laitinen RA, Teeri TH. Activation of anthocyanin biosynthesis in Gerbera hybrida (Asteraceae) suggests conserved protein-protein and protein-promoter interactions between the anciently diverged monocots and eudicots. Plant Physiol. 2003;133:1831-42.

8. Zhang Y, Butelli E, Martin C. Engineering anthocyanin biosynthesis in plants. Curr Opin Plant Biol. 2014;19:81-90.

9. Noda N, Aida R, Kishimoto S, Ishiguro K, Fukuchi-Mizutani M, Tanaka Y, et al. Genetic engineering of novel bluer-colored chrysanthemums produced by accumulation of delphinidin-based anthocyanins. Plant Cell Physiol. 2013;54:1684-95.

10. Noda N, Yoshioka S, Kishimoto S, Nakayama M, Douzono M, Tanaka Y, et al. Generation of blue chrysanthemums by anthocyanin B-ring hydroxylation and glucosylation and its coloration mechanism. Sci Adv. 2017;3:e1602785.

11. Schwinn K, Venail J, Shang Y, Mackay S, Alm V, Butelli E, et al. A small family of MYB-regulatory genes controls floral pigmentation intensity and patterning in the genus Antirrhinum. Plant Cell. 2006;18:831-51.

12. Schwinn KE, Boase MR, Bradley JM, Lewis DH, Deroles SC, Martin CR, et al. MYB and bHLH transcription factor transgenes increase anthocyanin pigmentation in petunia and lisianthus plants, and the petunia phenotypes are strongly enhanced under field conditions. Front Plant Sci. 2014;5:603.

13. Butelli E, Titta L, Giorgio M, Mock HP, Matros A, Peterek S, et al. Enrichment of tomato fruit with health-promoting anthocyanins by expression of select transcription factors. Nat Biotechnol. 2008;26:1301-8.

14. Laitinen RA, Ainasoja M, Broholm SK, Teeri TH, Elomaa P. Identification of target genes for a MYB-type anthocyanin regulator in Gerbera hybrida. J Exp Bot. 2008:59:3691-703.

15. Yamagishi M, Toda S, Tasaki K. The novel allele of the LhMYB12 gene is involved in splatter-type spot formation on the flower tepals of Asiatic hybrid lilies (Lilium spp.). New Phytol. 2014;201:1009-20.

16. Li C, Qiu J, Yang G, Huang S, Yin J. Isolation and characterization of a R2R3MYB transcription factor gene related to anthocyanin biosynthesis in the spathes of Anthurium andraeanum (Hort.). Plant Cell Rep. 2016;35:2151-65. 
17. Chen K, Liu H, Lou Q, Liu Y. Ectopic expression of the grape hyacinth (Muscari armeniacum) R2R3-MYB transcription factor gene, MaAN2, induces anthocyanin accumulation in tobacco. Front Plant Sci. 2017;8:965.

18. Xie DY, Sharma SB, Wright E, Wang ZY, Dixon RA. Metabolic engineering of proanthocyanidins through co-expression of anthocyanidin reductase and the PAP1 MYB transcription factor. Plant J. 2006:45:895-907.

19. Zvi MM, Shklarman E, Masci T, Kalev H, Debener T, Shafir S, et al. PAP1 transcription factor enhances production of phenylpropanoid and terpenoid scent compounds in rose flowers. New Phytol. 2012;195:335-45.

20. Borevitz JO, Xia Y, Blount J, Dixon RA, Lamb C. Activation tagging identifies a conserved MYB regulator of phenylpropanoid biosynthesis. Plant Cell. 2000;12:2383-93.

21. Lou Q, Wang L, Liu H, Liu Y. Anthocyanin profiles in flowers of grape hyacinth. Molecules. 2017;22:688.

22. Mori S, Asano S, Kobayashi H. Analyses of anthocyanidins and anthocyanins in flowers of Muscari spp. Bulletin of the Faculty of Agriculture, Niigata University. 2002;55:13-8.

23. Lou Q, Liu Y, Qi Y, Jiao S, Tian F, Jiang L, Wang Y. Transcriptome sequencing and metabolite analysis reveals the role of delphinidin metabolism in flower colour in grape hyacinth. J Exp Bot. 2014;65:3157-64.

24. Zimmermann IM, Heim MA, Weisshaar B, Uhrig JF. Comprehensive identification of Arabidopsis thaliana MYB transcription factors interacting with R/B-like BHLH proteins. Plant J. 2004;40:22-34.

25. Singh R, Low E-TL, Ooi LC-L, Ong-Abdullah M, Nookiah R, Ting N-C, et al. The oil palm VIRESCENS gene controls fruit colour and encodes a R2R3-MYB. Nat Commun. 2014;5:4106

26. Schwinn KE, Ngo H, Kenel F, Brummell DA, Albert NW, McCallum JA, et al. The onion (Allium cepa L.) R2R3-MYB gene MYB1 regulates anthocyanin biosynthesis. Front. Plant Sci. 2016;7:1865.

27. Li QF, He JX. BZR1 interacts with HY5 to mediate brassinosteroid- and lightregulated cotyledon opening in Arabidopsis in darkness. Mol Plant. 2016;9:113-25

28. Xie XB, Li S, Zhang RF, Zhao J, Chen YC, Zhao Q, et al. The bHLH transcription factor MdbHLH3 promotes anthocyanin accumulation and fruit colouration in response to low temperature in apples. Plant Cell Environ. 2012;35:1884-97.

29. Xu W, Dubos C, Lepiniec L. Transcriptional control of flavonoid biosynthesis by MYB-bHLH-WDR complexes. Trends Plant Sci. 2015;20:176-85.

30. Li C, Qiu J, Ding L, Huang M, Huang S, Yang G, Yin J. Anthocyanin biosynthesis regulation of DhMYB2 and DhbHLH1 in Dendrobium hybrids petals. Plant Physiol Biochem. 2017;112:335-45.

31. Nakatsuka T, Sato K, Takahashi H, Yamamura S, Nishihara M. Cloning and characterization of the UDP-glucose:anthocyanin 5-O-glucosyltransferase gene from blue-flowered gentian. J Exp Bot. 2008;59:1241-52.

32. Bai Y, Pattanaik S, Patra B, Werkman JR, Xie CH, Yuan L. Flavonoid-related basic helix-loop-helix regulators, NtAn1a and NtAn1b, of tobacco have originated from two ancestors and are functionally active. Planta. 2011;234:363.

33. Baudry A, Heim MA, Dubreucq B, Caboche M, Weisshaar B, Lepiniec L. TT2, TT8, and TTG1 synergistically specify the expression of BANYULS and proanthocyanidin biosynthesis in Arabidopsis thaliana. Plant J. 2004;39:366-80.

34. Yamagishi M, Shimoyamada Y, Nakatsuka T, Masuda K. Two R2R3-MYB genes, homologs of Petunia AN2, regulate anthocyanin biosyntheses in flower tepals, tepal spots and leaves of asiatic hybrid lily. Plant Cell Physiol. 2010;51:463-74.

35. Chiou C-Y, Yeh K-W. Differential expression of MYB gene (OgMYB1) determines color patterning in floral tissue of Oncidium Gower Ramsey. Plant Mol Biol. 2008;66:379-88.

36. Hsu C-C, Chen Y-Y, Tsai W-C, Chen W-H, Chen H-H. Three R2R3-MYB transcription factors regulate distinct floral pigmentation patterning in Phalaenopsis spp. Plant Physiol. 2015;168:175-91.

37. Matus JT, Aquea F, Arce-Johnson P. Analysis of the grape MYB R2R3 subfamily reveals expanded wine quality-related clades and conserved gene structure organization across Vitis and Arabidopsis genomes. BMC Plant Biol. 2008;8:83.

38. Feller A, Machemer K, Braun EL, Grotewold E. Evolutionary and comparative analysis of MYB and bHLH plant transcription factors. Plant J. 2011;66(1):94-116.

39. Stracke R, Werber M, Weisshaar B. The R2R3-MYB gene family in Arabidopsis thaliana. Curr Opin Plant Biol. 2001;4(5):447-56.

40. Ma D, Constabel CP. MYB repressors as regulators of Phenylpropanoid metabolism in plants. Trends Plant Sci. 2019;24(3):275-89.
41. Quattrocchio F, Wing JF, Leppen HT, Mol JN, Koes RE. Regulatory genes controlling anthocyanin pigmentation are functionally conserved among plant species and have distinct sets of target genes. Plant Cell. 1993:5:1497-512.

42. Kiferle C, Fantini E, Bassolino L, Povero G, Spelt C, Buti S, Giuliano G, Quattrocchio F, Koes R, Perata P, et al. Tomato R2R3-MYB proteins SIANT1 and SIAN2: same protein activity. Different Roles PLoS One. 2015;10:e0136365.

43. Walker AR, Lee E, Bogs J, McDavid DA, Thomas MR, Robinson SP. White grapes arose through the mutation of two similar and adjacent regulatory genes. Plant J. 2007:49:772-85.

44. Spelt C, Quattrocchio F, Mol JN, Koes R. anthocyanin1 of petunia encodes a basic helix-loop-helix protein that directly activates transcription of structural anthocyanin genes. Plant Cell. 2000;12:1619-31.

45. Starkevic P, Paukstyte J, Kazanaviciute V, Denkovskiene E, Stanys V, Bendokas $V$, et al. Expression and anthocyanin biosynthesis-modulating potential of sweet cherry (Prunus avium L.) MYB10 and bHLH genes. PLoS One. 2015;10:e0126991.

46. Espley RV, Hellens RP, Putterill J, Stevenson DE, Kutty-Amma S, Allan AC. Red colouration in apple fruit is due to the activity of the MYB transcription factor, MdMYB10. Plant J. 2007:49:414-27.

47. Hichri I, Heppel SC, Pillet J, Leon C, Czemmel S, Delrot S, Lauvergeat V, Bogs $J$. The basic helix-loop-helix transcription factor MYC1 is involved in the regulation of the flavonoid biosynthesis pathway in grapevine. Mol Plant 2010;3:509-23.

48. Liu XF, Yin XR, Allan AC, Lin-Wang $K$, Shi YN, Huang YJ, Ferguson IB, Xu CJ, Chen KS. The role of MrbHLH1 and MrMYB1 in regulating anthocyanin biosynthetic genes in tobacco and Chinese bayberry (Myrica rubra) during anthocyanin biosynthesis. Plant Cell Tiss Org. 2013;115(3):285-98.

49. Dooner HK, Robbins TP, Jorgensen RA. Genetic and developmental control of anthocyanin biosynthesis. Annual Rev Genet. 1991;25:173-99.

50. Xu W, Grain D, Bobet S, Le Gourrierec J, Thevenin J, Kelemen Z, et al. Complexity and robustness of the flavonoid transcriptional regulatory network revealed by comprehensive analyses of MYB-bHLH-WDR complexes and their targets in Arabidopsis seed. New Phytol. 2014;202:132-44.

51. Feyissa DN, Lovdal T, Olsen KM, Slimestad R, Lillo C. The endogenous GL3, but not EGL3, gene is necessary for anthocyanin accumulation as induced by nitrogen depletion in Arabidopsis rosette stage leaves. Planta. 2009;230:747-54.

52. Ludwig SR, Bowen B, Beach L, Wessler SR. A regulatory gene as a novel visible marker for maize transformation. Science. 1990;247(4941):449-50.

53. Kim DH, Park S, Lee JY, Ha SH, Lim SH. Enhancing flower color through simultaneous expression of the B-Peru and mPAP1 transcription factors under control of a flower-specific promoter. Int J Mol Sci. 2018;19:309.

54. Pattanaik S, Kong Q, Zaitlin D, Werkman JR, Xie CH, Patra B, Yuan L. Isolation and functional characterization of a floral tissue-specific R2R3 MYB regulator from tobacco. Planta. 2010;231(5):1061-76.

55. Huang W, Khaldun AB, Lv H, Du L, Zhang C, Wang Y. Isolation and functional characterization of a R2R3-MYB regulator of the anthocyanin biosynthetic pathway from Epimedium sagittatum. Plant Cell Rep. 2016;35:883-94.

56. Tamura K, Stecher G, Peterson D, Filipski A, Kumar S. MEGA6: molecular evolutionary genetics analysis version 6.0. Mol Biol Evol. 2013;30:2725-9.

57. Yoo SD, Cho YH, Sheen J. Arabidopsis mesophyll protoplasts: a versatile cell system for transient gene expression analysis. Nat Protoc. 2007;2:1565-72.

58. Palapol Y, Ketsa S, Lin-Wang K, Ferguson IB, Allan AC. A MYB transcription factor regulates anthocyanin biosynthesis in mangosteen (Garcinia mangostana L.) fruit during ripening. Planta. 2009;229:1323-34.

59. Yin XR, Allan AC, Chen $\mathrm{KS}$, Ferguson IB. Kiwifruit EIL and ERF genes involved in regulating fruit ripening. Plant Physiol. 2010;153:1280-92.

60. Horsch RB. A simple and general method for transferring genes into plants. Science. 1985;227(4691):1229-31.

61. Qi Y, Lou Q, Li H, Yue J, Liu Y, Wang Y. Anatomical and biochemical studies of bicolored flower development in Muscari latifolium. Protoplasma. 2013;250:1273-81.

\section{Publisher's Note}

Springer Nature remains neutral with regard to jurisdictional claims in published maps and institutional affiliations. 